\title{
Towards multiband optical systems
}

\author{
Antonio Napoli ${ }^{1}$, Nelson Costa ${ }^{2}$, Johannes K. Fischer ${ }^{3}$, João Pedro ${ }^{2,4}$, Silvio Abrate ${ }^{5}$, \\ Nicola Calabretta ${ }^{6}$, Wladek Forysiak ${ }^{7}$, Erwan Pincemin ${ }^{8}$, Juan P. F-P. Gimenez ${ }^{9}$, \\ Chris Matrakidis ${ }^{10}$, Gunther Roelkens ${ }^{11}$, Vittorio Curri ${ }^{12}$ \\ ${ }^{1}$ Coriant R\&D GmbH, Germany; ${ }^{2}$ Coriant Portugal, Portugal; ${ }^{3}$ Frauhofer HHI, Germany; ${ }^{4}$ IT, IST, Portugal; \\ ${ }^{5}$ ISMB, Italy; ${ }^{6}$ TU/e, The Netherlands; ${ }^{7}$ AIPT, Aston University, UK; ${ }^{8}$ Orange Lab, France; ${ }^{9}$ Telefonica, Spain; \\ ${ }^{10}$ University of Peloponnese, Greece; ${ }^{11}$ Ghent University-IMEC, Belgium, ${ }^{12}$ Politecnico di Torino, Italy \\ antonio.napoli@coriant.com,nelson.costa@coriant.com, johannes.fischer@hhi.fraunhofer.de
}

\begin{abstract}
Multiband transmission is a valid option to significantly increase fiber capacity and efficiently utilize the available and deployed optical fiber infrastructure. In this contribution, we evaluate its challenges and possible implementation.
\end{abstract}

OCIS codes: (060.2330) Fiber optics communications; (060.1660) Coherent communications.

\section{Introduction}

The race to increase fiber capacity moved from static systems, employing intensity modulation direct-detection (IMDD), to advance elastic optical networks [1,2] where complex quadrature amplitude modulation (QAM) formats are transmitted by bandwidth variable transponders [3]. This significantly extended the fiber capacity, moving from $<1 \mathrm{~Tb} / \mathrm{s} /$ fiber with $10 \mathrm{~Gb} / \mathrm{s}$ IM-DD per channel to $>25 \mathrm{~Tb} / \mathrm{s} /$ fiber with $200 \mathrm{~Gb} / \mathrm{s}$ polarization multiplexing 16-QAM per channel. However, forecasts suggest that this capacity might not be sufficient to cope with the current growth of IP traffic, as we enter the Zettabyte era [4]. Furthermore, the imminent deployment of 5G networks and high-capacity access networks will further stress telecommunication infrastructures. To cope with this high demand for capacity, researchers proposed several solutions, mainly based on space division multiplexing (SDM), by transmitting over multi-fibers (MF) and/or multi-core (MC) and/or multi-mode (MM) fibers. In this contribution, we discuss a different approach, which aims at transmitting over the entire low-loss optical spectrum of single mode fibers (SMF), namely from $1260 \mathrm{~nm}$ to $1625 \mathrm{~nm}$. We name this as multiband (MB). The main objective of this article is to compare the advantages and shortcomings of MB and SDM, taking into account technology availability and possible applications.

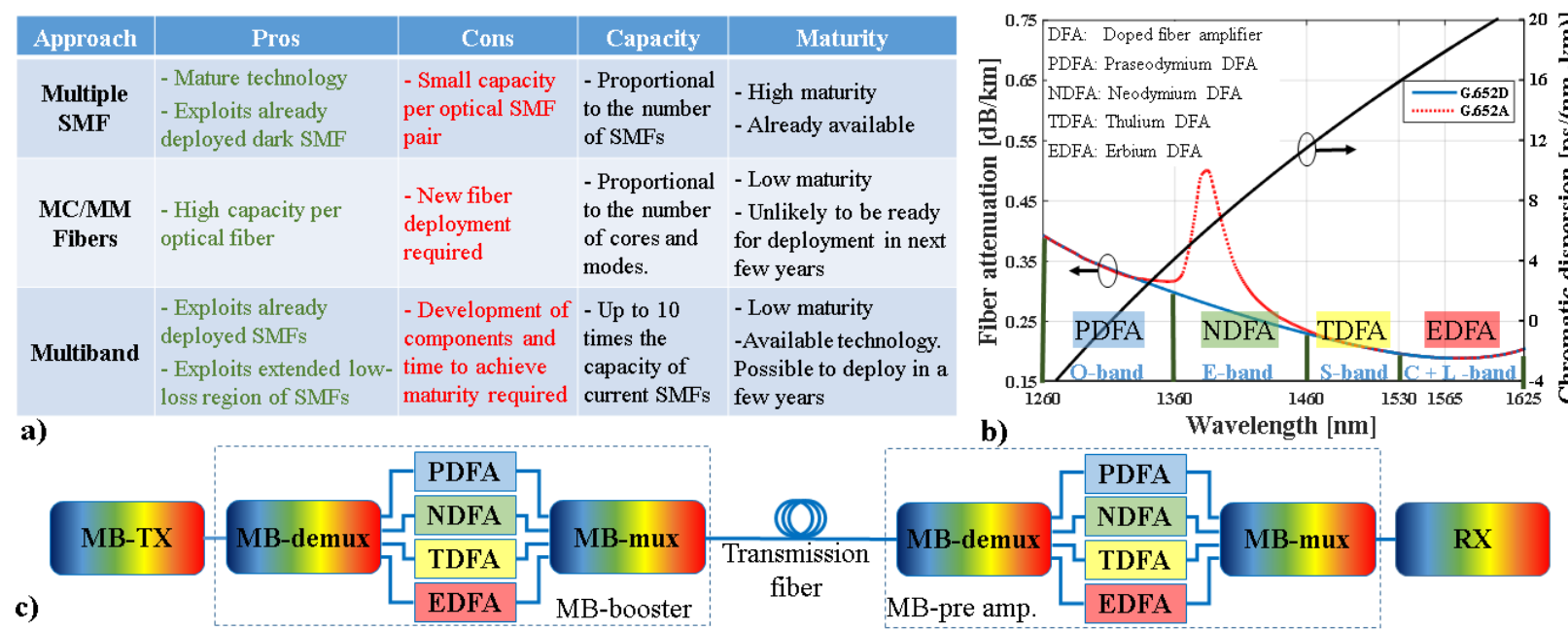

Fig. 1: (a): Comparison among the different solutions; (b): Fiber attenuation and dispersion parameters over the multiband SMF spectrum; (c): Multiband system and bandwidth requirements of the key-components.

\section{Solutions for capacity increase}

Large investments have been realized with the goal of extending the total capacity of optical systems. Within SDM, we can identify the following solutions: Multi\{-Fiber, -Mode, -Core\} and Fig. 1 1 a) compares them. Only MF can be already realized, for example by installing several parallel systems [5] over new fibers or by lighting up the existing 
dark fibers. However, this is a costly solution due to the multiplication of all network elements, and possibly requiring also the deployment or renting of additional optical fiber pairs, potentially entailing prohibitive costs. The MC and MM strategies have been widely investigated and numerous hero experiments have been reported. For instance, capacity in excess of $138 \mathrm{~Tb} / \mathrm{s}$ with MM [6] or up to $1 \mathrm{~Pb} / \mathrm{s}$ with MC [7] has been achieved. Such strategies would greatly contribute to address the capacity crunch, but they present several important limitations, as listed in Fig. 1 1 a). For example, they require the deployment of novel fibers, which is a very costly operation. Moreover, the transponders and also the digital signal processing units require a large amount of power because of the high-complexity MIMO equalizers. This makes them less suitable for the most relevant scenario for these solutions, i.e., short links. The last solution, MB, aims at exploiting spectral bands beyond the C-band, in order to maximize the return on investment of already deployed optical infrastructure which, in the case of Europe, consists almost entirely of optical cables without water peak [8]. This is showed in Fig. 11.b), where the five available bands (O, E, S, C, and L) present an attenuation coefficient below $0.4 \mathrm{~dB} / \mathrm{km}$. Naturally, MB still presents numerous challenges, as discussed in next section.

\section{Multiband approach, technology requirements and challenges}

Fig. 11 b) reports the attenuation and dispersion over wavelength of two exemplary fiber types: ITU-T G.652A (red) and G.652D (blue). The low-loss of G.652D across all bands (i.e. 1260 - $1625 \mathrm{~nm}$ ), could be exploited to increase the transmission bandwidth by several tens of THz up to $365 \mathrm{~nm}$ compared to C-band only systems [9]. Such a solution would be highly competitive against MF, MC and MM, in particular for metro and regional distances, where fiber losses (even in O-band) should be tolerable at the receiver. Fig. 1. (c) displays a simplified block diagram of a MB system for a point-to-point link, such as the ones for data-center interconnection. In this scenario, we envision that all network components must be developed. More precisely, the following elements must be covered: (I) the transceiver (laser, modulator, front-end, etc.) must be realized (in Indium Phosphide - InP or Silicon Photonics - SiPh [10]) possibly with the capability of being tunable over the entire spectrum. The DSP would likely remain (at least for short reach) similar to the existing ones; (II) the amplifiers and filters must be designed by utilizing the best doped material for each band. For instance, in Fig. 1 (b) we assume a possible choice for the rare earth materials used within the amplification stage. Other possibilities, such as Bismuth-DFA, Raman and semiconductor optical amplifiers (SOA) can also be considered. MB enables a pay-as-you-grow upgrade strategy, so that operators can install the additional bands only when required. Finally, MB transmission will need a sophisticated software-defined network (SDN) platform to dynamically and efficiently manage the enormous bandwidth. Such SDN platform requires a physical layer controller that provides an accurate yet quick quality-of-transmission estimator (QoT-E) that enables exploiting the differently performing bands [9] for transmitting, e.g., different classes of services. Such a QoT-E will need a feasible transmission model generalizing the ones that have been developed for the C-band. A first step in this direction has been presented in [11].

\section{Conclusions}

We discussed the great potential of MB transmission. We compared this solution against the ones commonly proposed, by evaluating the pros and cons. We conclude that MB transmission is a viable and realistic solution for the imminent deployment of next generation wireless infrastructure, although specific developments are still required.

Acknowledgment

A. Napoli would like to thank the German BMBF and grant no. 16KIS0487K (Celtic project SENDATE-FICUS) for partially funding this work. N. Costa, J. Pedro, J. K. Fischer and N. Calabretta would like to thank the EU 5G-PPP project METRO-HAUL grant no. 761727.

\section{References}

1. A. Napoli et al. "Next generation elastic optical networks: The vision of the European research project IDEALIST," IEEE Comm. Mag., vol. 53, no. 2, pp. 152-162, 2015.

2. V. López and L. Velasco. "Elastic Optical Networks," Springer International Publishing:, 2016.

3. N. Sambo et al. "Next generation sliceable bandwidth variable transponders," IEEE Comm. Mag., vol. 53, no. 2, pp. 163-171, 2015.

4. https://www.cisco.com/c/en/us/solutions/collateral/service-provider/visual-networking-indexvni/complete-white-paper-c11-481360.html

5. http://www.lightwaveonline.com/articles/2017/04/verizon-signs-1-05b-fiber-deal-with-corningto-support-wireless-broadband.html

6. J. van Weerdenburg et al. "138-Tb/s mode- and wavelength-multiplexed transmission over six-mode graded-index fiber," JLT, 2018.

7. T. Kobayashi et al., "1-Pb/s (32 SDM/46 WDM/768 Gb/s) C-band dense SDM transmission over 205.6-km...," paper Th5B.1, OFC, 2017.

8. https://www.fibre-systems.com/viewpoint/getting-most-currently-deployed-optical-fiberinfrastructure

9. S. Okamoto et al., "5-band (O, E, S, C, and L) WDM transmission with wavelength adaptive modulation format allocation," ECOC, 2016.

10. C. Doerr et al., "O, E, S, C, and L band silicon photonics coherent modulator/receiver," paper Th5C.4, OFC, 2016.

11. M. Cantono et al. "Modelling the impact of SRS on NLI generation in commercial equipment...," Paper M1D.2, OFC, 2018. 